\title{
Reconstruction of Large Orbital Floor Defect Caused by Maxillary Sinus Mucocele
}

\author{
Seung Bum Pyo, \\ Jin Kyung Song, \\ Hong Sil Ju, \\ Seong Yoon Lim \\ Department of Plastic and Reconstructive \\ Surgery, Hanil General Hospital, Seoul, Korea
}

No potential conflict of interest relevant to this article was reported.

\begin{abstract}
Maxillary sinus mucocele can occur due to many medical factors such as chronic infection, allergic sinonasal disease, trauma, and previous surgery. However, it occurs mainly after Caldwell-Luc operation, usually more than 10 years after surgery. There are a few cases of maxillary sinus mucocele with ocular symptoms. Also, a case causing ocular symptoms because of invasion to the orbital floor is rare. Therefore, we report a case of a 55-year-old male patient who underwent Caldwell-Luc operation about 30 years ago. Then, symptoms such as exophthalmos, diplopia, and visual disturbance developed suddenly 3 months prior to admission. Computed tomography showed a cyst invading the orbital floor which resulted in eyeball deviation. The orbital floor defect measured approximately $2.5 \times 3.3 \mathrm{~cm}$. Maxillary sinus mucocele was removed through an endoscopic approach. After this, we reconstructed the orbital floor through a subciliary incision. Observation was carried out after three years, and ocular symptoms such as diplopia and exophthalmos did not recur.
\end{abstract}

Keywords: Maxillary sinus / Mucocele / Exophthalmos

\section{INTRODUCTION}

Postoperative maxillary sinus mucocele develops following obstruction of the sinus ostium after Caldwell-Luc operation, usually more than 10 years after surgery [1]. Maxillary sinus mucocele is a benign tumor, but it slowly increases in size, resulting in erosion and remodeling of the surrounding bone. As a result, enlarged mucocele can causes nasal obstruction, facial asymmetry, visual changes, and dental problems [2]. Bone destruction caused by mucocele is rare. In most cases, it is generally easier for the mucocele to invade the anterior and medial walls of the maxillary sinus with bone erosion. Also, the occurrence of ocular symptoms, such as deviated eyeball, exophthalmos, visual field defect, decreased visual acuity, and diplopia, is rare. Minimal bony erosion caused by an expanding cyst has not been reconstructed. Howev-

\section{Correspondence: Jin Kyung Song}

Department of Plastic and Reconstructive Surgery, Hanil General Hospital, 308 Uicheonro, Dobong-gu, Seoul 01450, Korea

E-mail: cococoeye@gmail.com

Received May 31, 2017 / Revised September 8, 2017 / Accepted September 8, 2017 er, reconstruction of the orbital floor is required if the defect area is wide following cyst removal [3].

We experienced a case of large maxillary sinus mucocele with ocular symptoms. In this study, we would like to report the result of postoperative maxillary sinus mucocele with ocular symptoms caused by a large defect in the orbital floor.

\section{CASE REPORT}

A 55-year-old male patient presented with left unilateral exophthalmos, diplopia, and visual impairment (Fig. 1). These symptoms developed three months before the hospital visits. The patient had a history of hypertension, tuberculosis, cerebral infarction, angina, multi-infarct dementia, glaucoma, and radical surgery for maxillary sinusitis performed about 30 years ago, but he did not have any recent history of trauma or infection.

Computed tomography (CT) and magnetic resonance imaging (MRI) showed a cyst formed in the maxillary sinus, invading the orbital floor which resulted in eyeball deviation. Orbital floor 


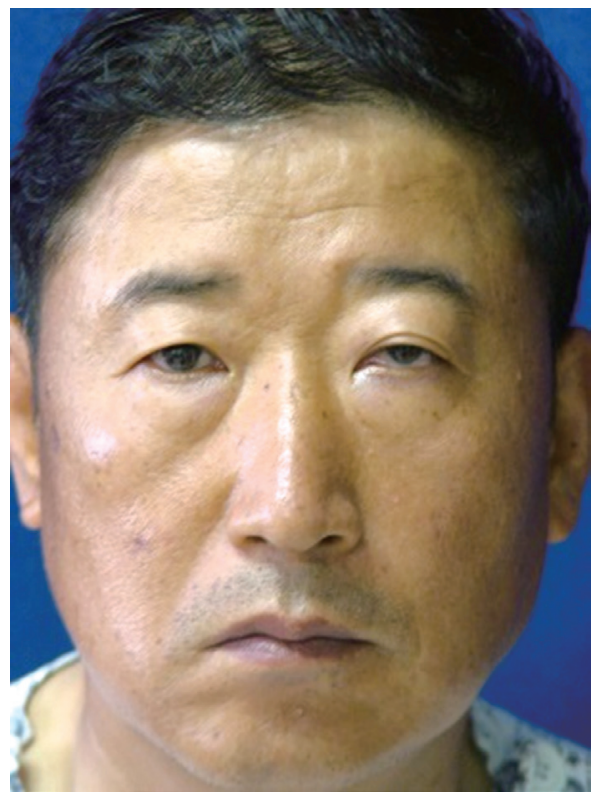

Fig. 1. Preoperative photograph of the patient shows left exophthalmos with ptosis.

defect was caused by the enlarged cyst, and the lesion measured approximately $2.5 \times 3.3 \mathrm{~cm}$. Also, the lateral and upward deviation of the left eyeball was found in the imaging test (Fig. 2).

The operation was performed in cooperation with the Department of Otorhinolaryngology, and the maxillary sinus mucocele was removed through an endoscopic approach. After this, we reconstructed the orbital floor through a subciliary incision. Then, we completed the operation with absorbable plate fixation on the orbital floor (Fig. 3). The biopsy from the cyst was consistent benign cyst, corresponding with the findings of the maxillary sinus mucocele (Fig. 4). After surgery, symptoms such as exophthalmos, diplopia, and visual disturbance were improved (Fig. 5). Postoperative CT scan showed a stably reconstructed orbital floor (Fig. 6).

The patient was discharged 7 days after the operation without any complications. Two years after surgery, when the patient blew his nose too hard, a left periocular swelling developed. Then, CT showed thickened mucosa of the sinus besides the nose and swelling of nasal concha. However, there was no invasion of the orbital wall, and reconstruction of the orbital floor was maintained stably (Fig. 7). Three years after surgery, we observed none of the sequelae such as diplopia and exophthalmos (Fig. 8).
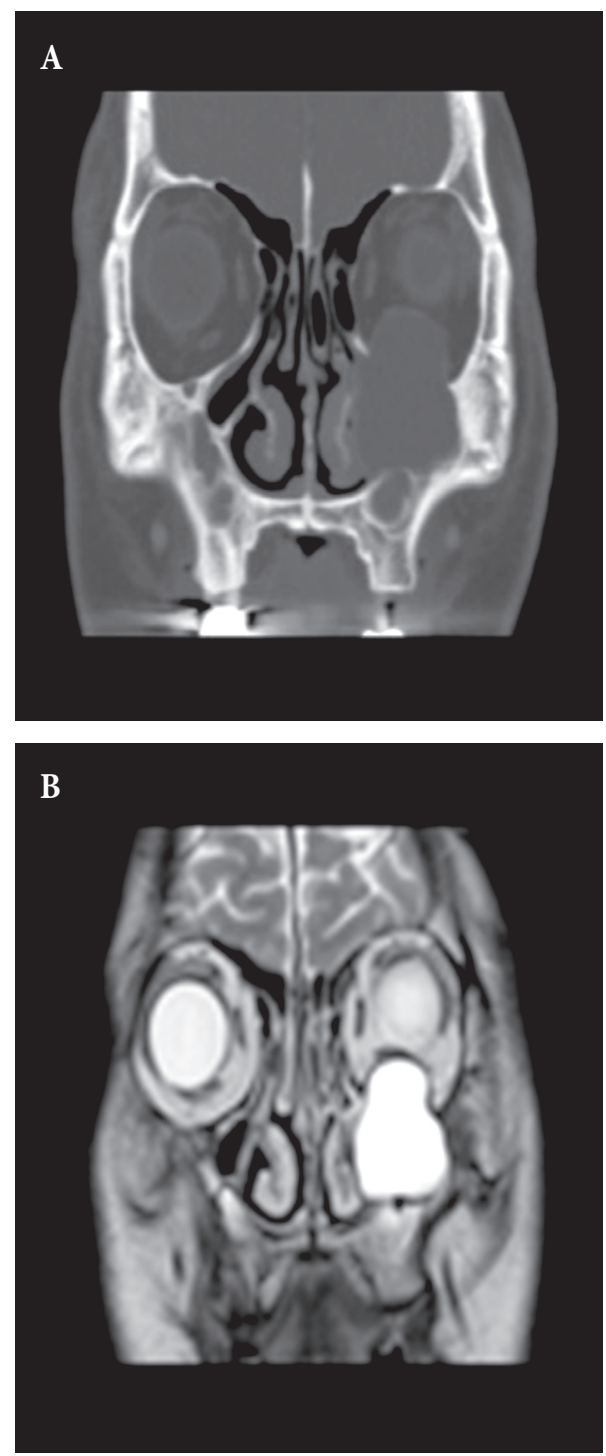

Fig. 2. Preoperative computed tomography image (A) and magnetic resonance imaging (B) shows a maxillary sinus cyst that invaded the orbital floor, resulting in eyeball deviation.

\section{DISCUSSION}

Maxillary sinus mucocele is a benign lesion which is relatively rare, and it accounts for about $10 \%$ of all paranasal sinus mucoceles. It is known that the incidence of maxillary sinus mucocele is greater in Korea and Japan than in other countries, such as Europe and USA. This is because high incidence of the cyst in Japan coincided with widespread use of the Caldwell-Luc operation for 


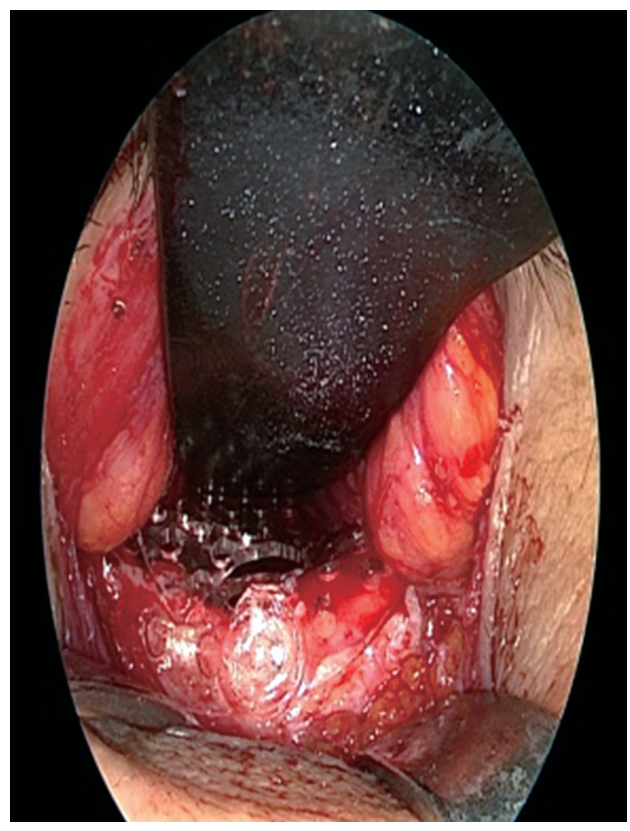

Fig. 3. Intraoperative endoscopic image shows reconstruction of the orbital floor via a subciliary incision with absorbable plate and screw.

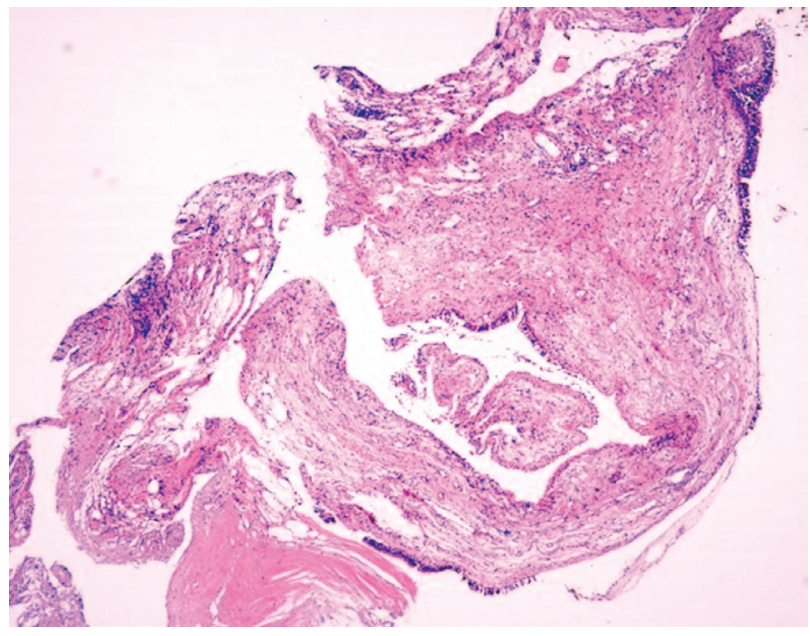

Fig. 4. Histologic examination of the intraoperative biopsy is corresponding with benign cyst $(\mathrm{H} \& \mathrm{E}, \times 40)$.

treating maxillary sinusitis [1]. In Japan, approximately 20 percent of the patients who underwent radical maxillary sinus surgery suffered from maxillary sinus mucocele [4]. In Korea, there was one study in the area of plastic surgery that showed occurrence of maxillary sinus mucocele after reduction malarplasty. However, the cyst expanded to the anterior and lateral walls of the sinuses, and the impact on the orbital floor was not significant [5].

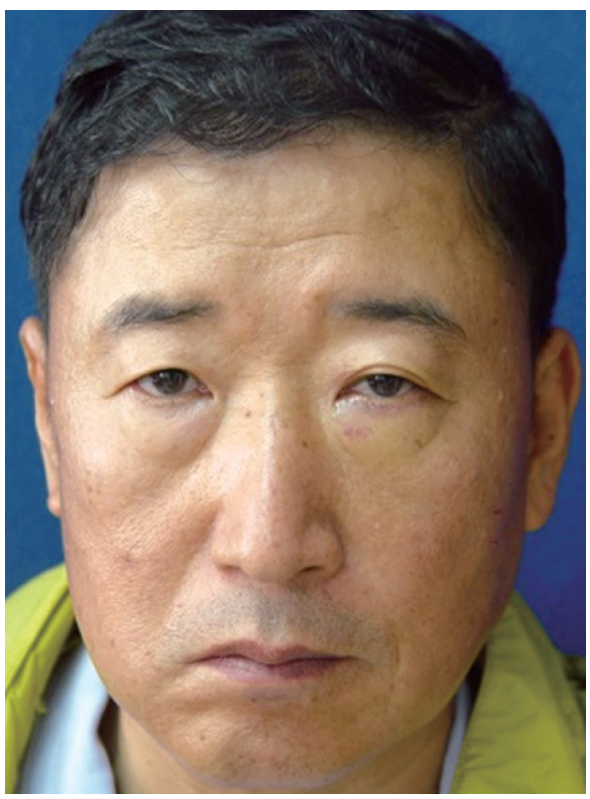

Fig. 5. Postoperative photograph of the patient shows that exophthalmos was completely corrected.

Many medical factors cause maxillary sinus mucocele, such as chronic infection, allergic sinonasal disease, trauma, previous surgery, and previous radiotherapy [6]. The symptoms of maxillary sinus mucocele develop gradually after blockage of a sinus ostium. In the early stages of its occurrence, mucocele may be asymptomatic for years. As it enlarges adequately and compresses the wall of the maxillary sinus, bone destruction can occur. Then, the patient complains of cheek swelling and unpleasant sensation on the cheek. At the end of the stage, the orbital floor is invaded, resulting in ocular symptoms such as exophthalmos, decreased visual acuity, diplopia, and strabismus. Maxillary sinus mucocele is commonly diagnosed by CT. CT is highly valued because of its diagnostic ability in detecting bone changes such as bone erosion [7].

Because the cyst can cause complications and permanent damage to the surrounding structure, surgical treatment may be needed. Recently, an endoscopic approach was performed and it provided a good result [7]. Endoscopic removal of the mucocele can causes a defect of approximately $1 \mathrm{~cm}^{2}$ in the orbital floor. In general, treatment may be indicated for repair of the orbital wall, if the defect size in the orbital floor is greater than $1 \mathrm{~cm}^{2}[8]$.

The patient's CT and MRI showed a cyst invading the orbital floor which resulted in exophthalmos, diplopia, and visual distur- 


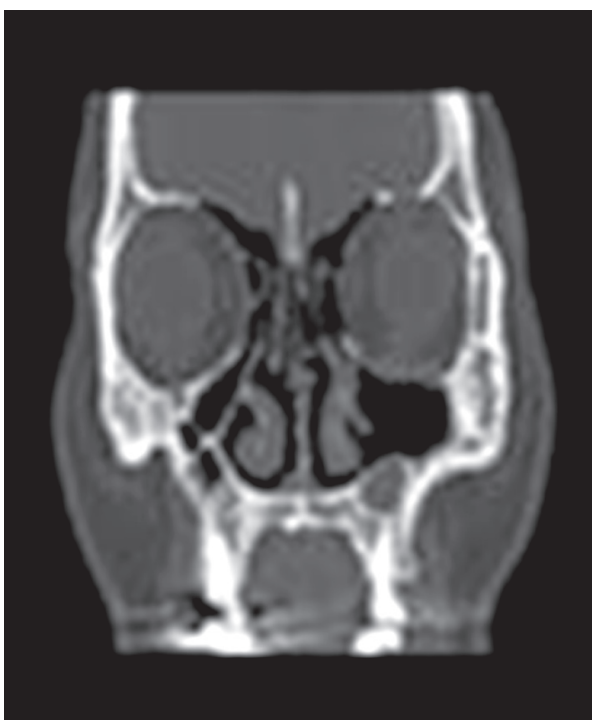

Fig. 6. Postoperative computed tomography scan shows a stably reconstructed orbital floor.
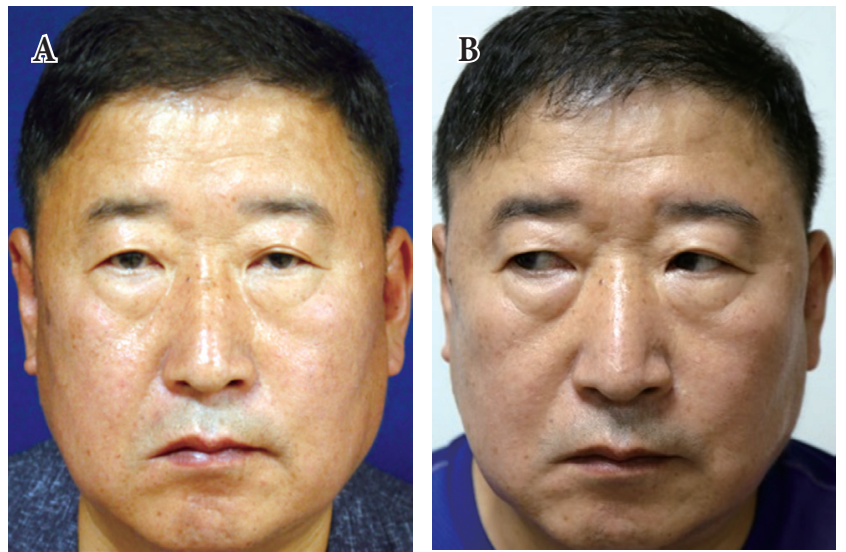

Fig. 8. Postoperative photograph of the patient at 3 years showed no ocular complication such as exophthalmos, diplopia, and limitation of ocular movement. (A) Frontal view. (B) Rightward movement of the eyes. (C) Leftward movement of the eyes. (D) Upward movement of the eyes.

bance. The cyst was removed via the endoscopic approach method for preventing permanent damage, and reconstruction with an absorbable plate was performed for large defect in the orbital floor. After surgery, the patient's symptoms were improved.

We present a method to effectively manage rare cases with a large defect in the orbital floor following cyst expansion caused by maxillary sinus mucocele. After the cyst was removed, an absorbable plate was used for reconstructing the orbital floor to prevent enophthalmos. As a result, the patient's symptoms were relieved,
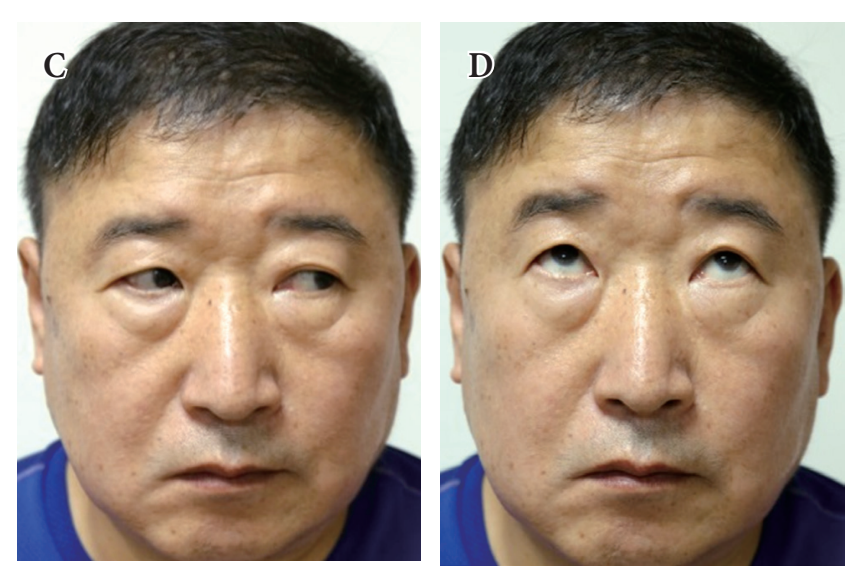

Fig. 7. Postoperative computed tomography views at 2 years showed thickened mucosa of the maxillary sinus, but reconstruction of the orbital floor was maintained stably. and postoperative complications such as eyeball deviation, diplopia, and enophthalmos did not recur during 3 years. In our case, the patient was first diagnosed with only ocular symptoms, and he visited the Department of Ophthalmology. We suggest that it is necessary to obtain the past history of the patient's previous facial bone fracture who complained about only ocular symptoms such as diplopia and exophthalmos for identifying the possibility of maxillary sinus mucocele. 


\section{PATIENT CONSENT}

The patient provided written informed consent for the publication and the use of their images.

\section{REFERENCES}

1. Costan VV, Popescu E, Stratulat SI. A new approach to aesthetic maxillofacial surgery: surgical treatment of unilateral exophthalmos due to maxillary sinus mucocele. J Craniofac Surg 2013;24:914-6.

2. Durr ML, Goldberg AN. Endoscopic partial medial maxillectomy with mucosal flap for maxillary sinus mucoceles. Am J Otolaryngol 2014;35:115-9.

3. Choi IS, Kim JS, Jun BH. Two cases of postoperative cheek cyst with exophthalmos. Korean J Otolaryngol-Head Neck Surg 2005;48:267-70.

4. Lee JH, Huh KH, Yi WJ, Heo MS, Lee SS, Choi SC. Bilateral postoperative maxillary cysts after orthognathic surgery: a case report. Imaging Sci Dent 2014;44:321-4.

5. Kim HI, Roh SG, Lee NH, Yang KM. Giant maxillary mucocele occurring after reduction malarplasty. J Craniofac Surg 2012;23:el23-4.

6. Abdel-Aziz M, El-Hoshy H, Azooz K, Naguib N, Hussein A. Maxillary sinus mucocele: predisposing factors, clinical presentations, and treatment. Oral Maxillofac Surg 2017;21:55-8.

7. Jang TY, Lee SJ, Kim KT, Choi YS. Management of postoperative mucocele of the maxillary sinus through endoscopic approach. Korean J Otolaryngol-Head Neck Surg 2005;48:314-9.

8. Jung S, Oh HK, Park HJ, Kook MS. Surgical enucleation of the mucocele on the inferior orbit using transantral approach. J Craniofac Surg 2015;26:e546-7. 